\title{
Q qumestão
}

\section{Análise bibliométrica da produção científica brasileira e do nordeste em Biotecnologia}

\author{
José Erivaldo Bezerra de Freitas \\ Doutorando; Universidade Federal do Ceará, Fortaleza, CE, Brasil; \\ selma@ufc.br \\ Selma Elaine Mazzetto \\ Doutora; Universidade Federal do Ceará, Fortaleza, CE, Brasil; \\ selma@ufc.br \\ Roniberto Morato do Amaral \\ Doutor; Universidade Federal de São Carlos, São Carlos, SP, Brasil; \\ roniberto@ufscar.br \\ Leandro Innocentini Lopes de Faria \\ Doutor; Universidade Federal de São Carlos, São Carlos, SP, Brasil; \\ leandro@ufscar.br \\ Daniel Rodrigo Leiva \\ Doutor; Universidade Federal de São Carlos, São Carlos, SP, Brasil; \\ daniel.leiva@ufscar.br \\ Douglas Henrique Milanez \\ Doutor; Universidade Federal de São Carlos, São Carlos \\ douglasmilanez@yahoo.com.br
}

\begin{abstract}
Resumo: A Biotecnologia consolida-se como um tema recorrentemente tratado como estratégico para o desenvolvimento do Brasil. Neste contexto, há necessidade de se investigar o impacto das políticas públicas de ciência e tecnologia, como os Planos Plurianuais e a Estratégia Nacional de Ciência e Tecnologia, que visam estimular a pesquisa científica na área. O objetivo deste artigo foi elaborar e analisar um conjunto de indicadores bibliométricos sobre a produção científica em Biotecnologia. O método utilizado compreendeu o uso da Bibliometria como técnica de análise de informações. A amostra analisada foi de 517.569 registros bibliográficos referentes à produção científica sobre Biotecnologia indexada na base de dados Web of Science, no período de 2001 a 2015. Os resultados alcançados compreenderam indicadores de produção e de colaboração científica nacional e internacional e apontam para o aumento da contribuição do Brasil frente à produção científica mundial neste assunto e o crescimento da importância das pesquisas realizadas pelas instituições da região nordeste do Brasil, bem como a colaboração entre elas. Conclui-se que a implementação de políticas públicas em ciência e tecnologia visando à redução das desigualdades regionais tem surtido efeito na área de Biotecnologia.
\end{abstract}

Palavras-chave: Biotecnologia. Indicadores de Ciência \& Tecnologia. RENORBIO. Bibliometria. 


\section{Introdução}

A Biotecnologia é uma área de pesquisa estratégica para o desenvolvimento científico, tecnológico, econômico, ambiental e social do Brasil, devido à biodiversidade que caracteriza os vários biomas espalhados pelo país (BRASIL, 2012; BRASIL, 2015a). De acordo com o Ministério da Saúde, a Biotecnologia refere-se a "[...] qualquer aplicação tecnológica que utilize sistemas biológicos, organismos vivos ou seus derivados, para fabricar ou modificar produtos ou processos para utilização específica.” (BRASIL, 2016a).

Assim como em outros países, o Brasil estabeleceu, em sua Estratégia Nacional de Ciência, Tecnologia e Inovação (ENCTI), o direcionamento do fomento em pesquisa para áreas e tecnologias estratégicas, entre as quais está a Biotecnologia (BRASIL, 2012). Desde 2001, foi instituído pelo Governo Federal, por meio da Lei $n^{\circ} 10.332$ (BRASIL, 2001a), o Fundo Setorial de Biotecnologia - CT-Biotecnologia, com objetivo de incentivar o desenvolvimento científico e tecnológico brasileiro em Biotecnologia e recursos genéticos. Naquela época, as diretrizes estratégicas indicaram como desafio para o país na área (CENTRO DE GESTÃO E ESTUDOS ESTRATÉGICOS, 2002): criação e fortalecimento de empresas de base biotecnológica; uso da biodiversidade; fortalecimento da infraestrutura para a Biotecnologia; biossegurança e bioética; formação de recursos humanos; atividades de cooperação internacional; atividades de monitoramento, acompanhamento, gestão e prospecção.

No Brasil, o Estado é reconhecidamente o organismo responsável pela alocação dos recursos em ciência e tecnologia, assim, a necessidade de avaliação da atividade científica torna-se fundamental, pois os investimentos destinados à ciência são limitados e competem com outros investimentos dos setores públicos (DAVYT; VELHO, 2000). As iniciativas de avaliação da ciência se concentram na elaboração de metodologias apropriadas para a construção de indicadores, que surgem da medição dos insumos (inputs) e resultados (outputs) da instituição científica, país ou região geográfica. Sendo que insumo seria a combinação de fatores que viabilizam a produção de determinada quantidade de 
resultado científico (SPINAK, 1998). Portanto, a avaliação é parte do processo de construção do conhecimento científico, através dela que se define o rumo da ciência e das instituições vinculadas (DAVYT; VELHO, 2000). Desse modo, as avaliações quantitativas das publicações científicas, através dos estudos métricos (FARIA et al., 2010; FREEMAN; SOETE, 2009; GOKHBERG, 2013; MOED; GLÄNZEL; SCHMOCH, 2004; OECD, 2007; OKUBO, 1997; SPINAK, 1998), podem apontar os caminhos, por exemplo, para a alocação dos recursos destinados à atividade científica tecnológica (GREGOLIN et al., 2005).

Nesse contexto, a Biotecnologia tem sido objeto de diversos estudos métricos em Ciência da Informação envolvendo a elaboração e análise de indicadores bibliométricos de publicações científicas e de patentes. Há estudos que investigam de maneira ampla os padrões da produção científica internacional em Biotecnologia, com atenção à evolução temporal, temática abordada, e distribuição nacional das publicações (GLANZEL et al., 2003). Outros estudos focam aspectos mais específicos, como as conexões existentes entre ciência e tecnologia (HUANG; YANG; CHEN, 2015) e a importância da colaboração internacional para a pesquisa na área (PAYUMO; SUTTON, 2015). Há inclusive estudos que analisam a qualidade de análises bibliométricas anteriores na área de Biotecnologia (DALPÉ, 2002). No âmbito brasileiro, há estudo sobre as interações que ocorrem entre ciência e tecnologia nessa área (MOURA; CAREGNATO, 2010). E ainda, sobre quais motivos que levam os pesquisadores a desenvolver pesquisa em Biotecnologia e quais são os critérios utilizados para a determinação de parcerias e divisão da coautoria e coinvenção (MOURA, 2012).

Porém, considerando a importância da Biotecnologia para o Brasil, se faz necessário investigar o impacto das políticas públicas de ciência e tecnologia, envolvendo essa temática no Brasil, em especial para a região nordeste do país, que têm merecido especial atenção pelas iniciativas governamentais, com vistas à diminuição das assimetrias, quanto ao desenvolvimento científico e tecnológico, provocado pela concentração das Instituições de Ciência e Tecnologia (ICT) nas regiões Sul e Sudeste do Brasil. 
Visando contribuir para o desenvolvimento científico, tecnológico, econômico, ambiental e social do Brasil, o objetivo deste artigo foi elaborar e analisar um conjunto de indicadores bibliométricos sobre a produção científica em Biotecnologia, que poderá ser utilizado no suporte às políticas públicas, contribuindo para o planejamento e acompanhamento de programas de pesquisa, entre outras iniciativas. O método utilizado compreendeu o uso da Bibliometria como técnica de análise de informações (FARIA et al., 2010; FREEMAN; SOETE, 2009; GOKHBERG, 2013; MOED; GLÄNZEL; SCHMOCH, 2004; OECD, 2007; OKUBO, 1997; SPINAK, 1998) e a amostra analisada compreendeu 517.569 registros bibliográficos referentes à produção científica sobre Biotecnologia indexada na base de dados Web of Science, no período de 2001 a 2015.

\section{Biotecnologia e indicadores de produção científica}

A área de Biotecnologia tem enfrentado significativos desafios, pois, se representa expressivas oportunidades de desenvolvimento nos campos científico, tecnológico, econômico, social e ambiental, também é objeto de polêmicas relativas à biossegurança, tratada na Lei $\mathrm{N}^{\circ} 11.105$ de 2005, chamada de Lei de Biossegurança, e ao acesso ao patrimônio genético e ao conhecimento tradicional, tratados previamente pela Medida Provisória No 2.186-16/2001, suplantada em 2015 pela Lei No 13.123, conhecida como Lei da Biodiversidade (BRASIL, 2001b, 2005, 2015).

A legislação, a título de resguardar a biodiversidade e o conhecimento tradicional de populações sobre o uso desses recursos, impôs aos pesquisadores, até 2015, a necessidade de obtenção de autorizações para uso dos recursos naturais em pesquisa, inclusive impedindo o patenteamento de tecnologias eventualmente oriundas de pesquisas realizadas sem as devidas autorizações. Com a entrada em vigor da Lei de Biodiversidade em 2015, as requisições de autorização foram substituídas por registros de atividades de pesquisa, simplificando o acesso dos pesquisadores ao material genético.

Polêmicas à parte, a Biotecnologia é um tema recorrentemente tratado como estratégico no planejamento governamental e nas agências de fomento. É 
o caso, por exemplo, dos Planos Plurianuais lançados a cada quatro anos que estabelecem as diretrizes, objetivos e metas a serem seguidos pelos Governos Federal, Estadual ou Municipal. No Plano Plurianual de 2012-2015, a Biotecnologia é citada em uma meta estabelecida pelo Ministério de Ciência e Tecnologia e Inovação, que visava promover pesquisa, desenvolvimento tecnológico em áreas estratégicas de caráter transversal, ao lado da nanotecnologia e novos materiais. No mesmo plano, coube ao Ministério de Agricultura, Pecuária e Abastecimento, fomentar a inovação e disseminar a cultura de Propriedade Intelectual em vários temas, dentre os quais a Biotecnologia (BRASIL, 2016a). Já no Plano Plurianual 2016-2019, a Biotecnologia aparece juntamente como um subtema da bioeconomia, ao lado de fármacos, medicamentos e bioprodutos, e a meta, atribuída ao Ministério de Ciência, Tecnologia e Inovação, envolve, além de promover políticas públicas de pesquisa, minimizar as assimetrias regionais (BRASIL, 2016b).

A redução dessas assimetrias, assim como a ampliação da formação de recursos humanos especializados na agregação de valor à biodiversidade nacional, são ações a serem priorizadas segundo a Proposta de Estratégia Nacional de Ciência, Tecnologia e Inovação, o que tem sido buscado pelo fortalecimento das redes regionais de pesquisa em Biotecnologia (RENORBIO, BIONORTE e Rede Pró-Centro-Oeste) voltadas para esse fim (BRASIL, 2015a). Outra abordagem tem sido a ampliação da base de pesquisa científica e tecnológica em temas ligados à Biotecnologia, com investimentos em laboratórios regionais para disponibilizar equipamentos necessários ao desenvolvimento de pesquisas nessa área nas várias regiões do país (CENTRO DE GESTÃO E ESTUDOS ESTRATÉGICOS, 2002).

Nesse contexto, a partir de discussões que remontam ao ano de 1998, surgiu o programa Rede Nordeste de Biotecnologia (RENORBIO), em 2003, com a proposta de integrar a região no tema Biotecnologia. Resumidamente, os principais objetivos da rede incluem acelerar o processo de desenvolvimento da região com a formação de recursos humanos, incentivo à pesquisa científica e tecnológica, fomento a atividades que promovam inovação no setor privado e melhoria da qualidade de vida de sua população (RENORBIO, 2016). 
Desde o início do processo de institucionalização das políticas públicas de ciência e tecnologia do século vinte até os dias de hoje, as formas de avaliação, as bases conceituais, a estrutura organizacional, os instrumentos de financiamento são comuns aos países que implantaram políticas para estimular a produção de conhecimento científico e tecnológico. Entretanto, cada país aloca recursos com base em critérios ajustados ao seu próprio contexto e tem estruturas diversas de gestão pública, compreendendo políticas de ciência e tecnologia e órgãos de financiamento diferenciados (VELHO; SOUZA-PAULA, 2008).

Os indicadores são utilizados pelos governos e pelas agências de fomento no planejamento, execução e acompanhamento das políticas públicas em ciência e tecnologia, e, também, pela comunidade científica, como forma de compreender o sistema no qual ela está inserida. Já as empresas buscam compreender as contingências científicas e tecnológicas do seu ambiente competitivo. Assim, os indicadores são um meio para compreender e avaliar as dinâmicas das atividades científicas e tecnológicas e sua relação com a sociedade (VANTI, 2002). Gregolin et al., (2005, p. 5) definem indicadores “[...] como dados estatísticos usados para medir algo intangível, que ilustram aspectos de uma realidade multifacetada.”.

Apesar de lidar com um grande volume de informação, possibilitar o estabelecimento de um panorama amplo do objeto a ser investigado e evidenciar padrões que não são passíveis de serem observados de maneira qualitativa, a Bibliometria como técnica de análise de informações utilizada pelos estudos métricos da ciência, tem limitações que afetam a análise final, como: (1) o fato de não haver uma base de dados única que indexe toda produção científica mundial, tornando a análise amostral, além do fato da mesma ser dependente das características da base, pois nem toda informação presente no artigo é indexada; (2) a questão de que nem todos os resultados de pesquisa são publicados, além de, obviamente, as bases não acessarem ou contabilizarem as discussões científicas ricas que ocorrem entre pessoas; (3) não haver distinção efetiva da qualidade dos documentos, pois um artigo bastante citado, por exemplo, tem o mesmo valor de um artigo que nunca foi lido, embora publicado; (4) 
dificuldades de idioma, sendo que a maior parte das bases utilizadas para bibliometria estão em inglês; (5) desafios referentes à recuperação da informação, principalmente associadas ao desenvolvimento de expressões de busca que sejam robustas (FARIA et al., 2010; OKUBO, 1997). Apesar dessas limitações, os indicadores de produção científica têm contribuído de forma definitiva para a análise do desempenho e melhoria da eficiência dos sistemas nacionais de ciência, tecnologia e inovação.

\section{Método e desenvolvimento}

A metodologia empregada neste trabalho é descrita a seguir.

\subsection{Procedimento para recuperação dos dados bibliográficos de publicações científicas}

Indicadores bibliométricos da produção científica brasileira sobre Biotecnologia foram construídos a partir do conjunto de dados bibliográficos de publicações científicas indexadas na base Web of Science. A base foi selecionada por ser tradicionalmente utilizada em estudos bibliométricos, e por indexar um conjunto de periódicos de alta relevância para a pesquisa científica mundial (FARIA et al., 2010; GREGOLIN et al., 2005; MOED; GLÄNZEL; SCHMOCH, 2004; OKUBO, 1997). Os dados foram recuperados considerando o conjunto de publicações (artigos, artigos de revisão, notas e cartas) de periódicos classificados na área de pesquisa "Biotechnology \& Applied Microbiology" (Biotecnologia e Microbiologia Aplicada). Foram consideradas apenas as bases do Science Citation Index Expanded e Social Science Citation Index. A busca foi realizada no dia 22 de fevereiro de 2016 e recuperou 517.569 publicações na base, das quais 9.250 (1,8\%) possuem pelo menos um autor vinculado a uma instituição brasileira. Após as buscas, os dados bibliográficos das publicações foram coletados e armazenados em computador local para tratamento e elaboração dos indicadores. 


\subsection{Procedimento para elaboração dos indicadores de produção científica}

Depois da coleta e armazenamento, os dados sobre a produção científica em Biotecnologia foram importados no software de análise bibliométrica VantagePoint (v. 5.0), que foi utilizado como suporte para processamento da informação e elaboração dos indicadores de produção científica. Os seguintes indicadores foram elaborados:

a) número de publicações científicas do Brasil e do mundo;

b) contribuição percentual da produção científica brasileira;

c) contribuição percentual acumulada das regiões geográficas do Brasil entre 2001 e 2015, separadas em quinquênios (2001-2005, 2006-2010 e 2011-2015);

d) contribuição percentual acumulada das regiões geográficas do Brasil em quinquênios;

e) percentual de publicações do nordeste em colaboração com instituições do Brasil e do exterior em quinquênios;

f) rede de colaboração entre os estados do nordeste e outras regiões no período 2001-2015 - acumulado;

g) contribuição da RENORBIO em relação à produção científica do nordeste e do Brasil em quinquênios;

h) rede de colaboração das instituições integrantes da RENORBIO em quinquênios.

Para analisar a contribuição científica da RENORBIO, foram consideradas todas as publicações científicas efetuadas pelas instituições que a integram (RENORBIO, 2016). Embora não seja a estratégia de análise ideal, a separação das publicações efetuadas no âmbito da rede é desafiadora, visto que nem sempre os autores indicam se a publicação faz parte ou não da rede. Por outro lado, considera-se razoável a associação efetuada, visto que a rede integra boa parte das principais instituições de pesquisa da região nordeste (45 Instituições) e, consequentemente, estimula, direta ou indiretamente, as pesquisas em Biotecnologia quando no cumprimento do seu papel.

As redes de colaboração foram elaboradas com auxílio do software Gephi (v. 0.8.2) que possibilita a visualização dos elementos em uma rede 
bidimensional, além de fornecer métricas úteis para análise sociológica contextual, como densidade da rede, entre outros indicadores. Por fim, os gráficos foram construídos com auxílio do MS Excel (v. 2016).

\section{Resultados e discussão}

Os resultados do presente estudo são detalhados nesta seção.

\subsection{Pesquisas Científicas sobre Biotecnologia no Brasil e no nordeste}

A comunidade científica brasileira tem respondido positivamente à definição da Biotecnologia como uma área prioritária para a pesquisa no país. O número de publicações científicas brasileiras sobre o assunto tem aumentado consideravelmente desde o final da década 1980, conforme apresentado na Figura 1, passando de 2.502 artigos no período 2006-2010 para 3.849 no período 2011-2015. O número de publicações tem crescido consistentemente, quinquênio após quinquênio, apontando para uma tendência de crescimento para os próximos anos. O número crescente de publicações brasileiras em Biotecnologia indica aumento do esforço de pesquisa e da obtenção de avanços científicos nessa área prioritária. É importante destacar que o comportamento da produção científica brasileira está alinhado com a tendência mundial de crescimento acelerado do número de publicações na área, também mostrada na Figura 1.

Não apenas o número de publicações brasileiras em Biotecnologia tem aumentado, como também os pesquisadores brasileiros têm contribuído em uma parcela cada vez maior da produção científica mundial na área. No período 1991-1995, pesquisadores brasileiros eram coautores de apenas 0,7\% das publicações mundiais no assunto. No período 2006-2010, essa contribuição havia atingido 2,3\% e, no período 2011-2015, atingiu 2,8\%, como mostrado na Figura 2. O crescimento da contribuição brasileira para a produção científica mundial em Biotecnologia indica um fortalecimento da pesquisa nessa área no país.

É verdade que o crescimento da contribuição brasileira para a produção científica mundial seja um fenômeno comum a outras áreas de pesquisa, mas é 
importante ressaltar que a contribuição na área de Biotecnologia está sempre acima da contribuição média do país, considerando todas as áreas de pesquisa. Por exemplo, para o período 2011-2015, a contribuição média do Brasil para produção científica mundial em todas as áreas do conhecimento foi de 2,3\%, enquanto que para a área de Biotecnologia a contribuição foi de 2,8\%. Esses dados são consistentes com a literatura. Entre 2002 e 2006, o Brasil contribuiu em 1,7\% das publicações científicas mundiais considerando todas as áreas do conhecimento (FARIA et al., 2010) e em 1,9\% das publicações na área de Biotecnologia.

Figura 1 - Número de publicações científicas sobre Biotecnologia, no Mundo e no Brasil, por quinquênio, de 1971 a 2015

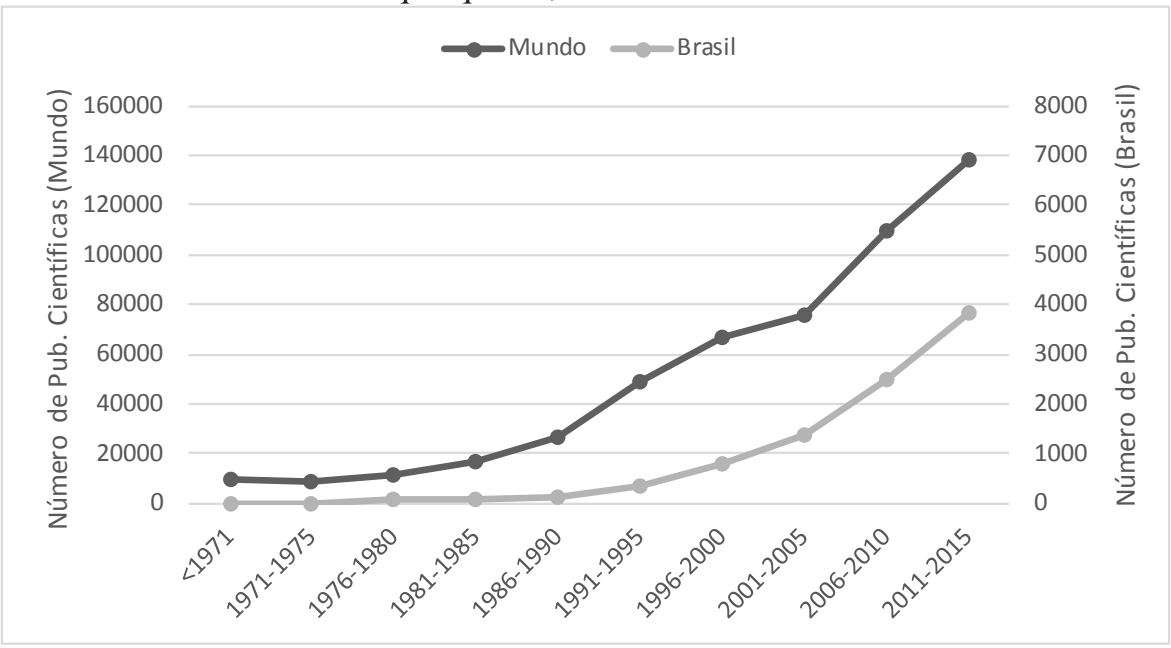

Fonte: Dados da pesquisa.

Figura 2 - Contribuição do Brasil para a produção científica mundial em Biotecnologia, 1971 a 2015

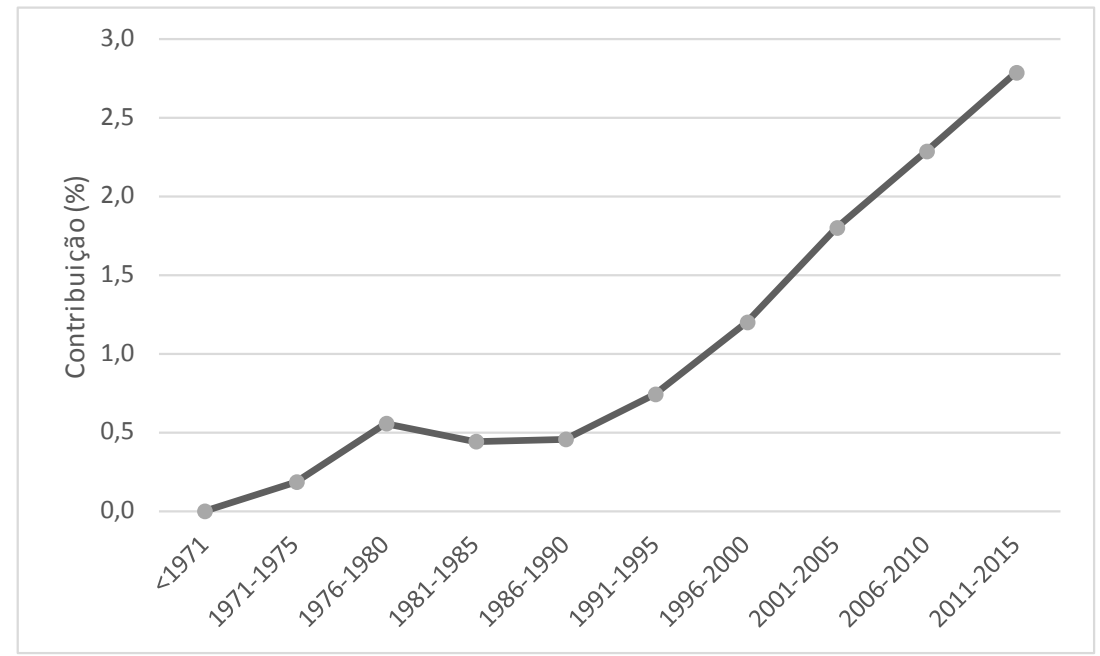

Fonte: Dados da pesquisa. 
De fato, desde 1982, o Brasil investe em projetos de pesquisa e na formação de recursos e infraestrutura voltada para Biotecnologia (CENTRO DE GESTÃO E ESTUDOS ESTRATÉGICOS, 2002), de forma mais intensa a partir dos anos 2000, através dos Planos Plurianuais que enxergam a Biotecnologia como área estratégica para a ciência, agricultura e saúde (BRASIL, 2016b), que são assuntos que têm apresentado crescimento expressivo no contexto científico nacional (FARIA et al., 2010). Mesmo com a aprovação da Lei de Biossegurança n 11.105 (BRASIL, 2005) e da Medida Provisória 2186-16 (BRASIL, 2001b), não houve queda na produção científica do Brasil, apesar da legislação em vigor no período estabelecer que a condução de atividades previstas na lei, entre as quais se encaixa a pesquisa científica, “[...] deverão requerer autorização para acesso ao patrimônio genético à Comissão Técnica Nacional de Biossegurança - CTNBio [...]” e ao Conselho de Gestão do Patrimônio Genético - CGEN.

Conforme mostra a Figura 3, entre 2001-2015, a região sudeste foi a região brasileira que mais publicou na área de Biotecnologia, contribuindo com pelo menos 76,5\% no período. Em segundo lugar, aparece a região sul, com $15,5 \%$, e a região nordeste, com 13,0\%. centro-oeste e norte ocupam as demais posições, com 8,25\% e 2,85\%, respectivamente. Em parte, o posicionamento das regiões geográficas brasileiras quanto à produção científica na área de Biotecnologia segue a classificação geral da produção científica nacional, considerando todos os temas (FARIA et al., 2010; GREGOLIN et al., 2005) e pode ser resultado de política de desconcentração da Ciência e Tecnologia no país, promovida pelo governo federal (CENTRO DE GESTÃO E ESTUDOS ESTRATÉGICOS, 2010).

Embora a região Sudeste tenha expressiva representatividade na área, sua contribuição tem diminuído ao longo dos períodos analisados, ao passo que o percentual de publicações das demais regiões, exceto a região Sul, tem aumentado nos últimos quinquênios. Essa tendência fornece indícios de que outras regiões brasileiras, em especial o nordeste, têm contribuído cada vez mais com as pesquisas sobre Biotecnologia desenvolvidas no cenário nacional. 
Figura 3 - Contribuição das diferentes regiões para a produção científica brasileira em Biotecnologia

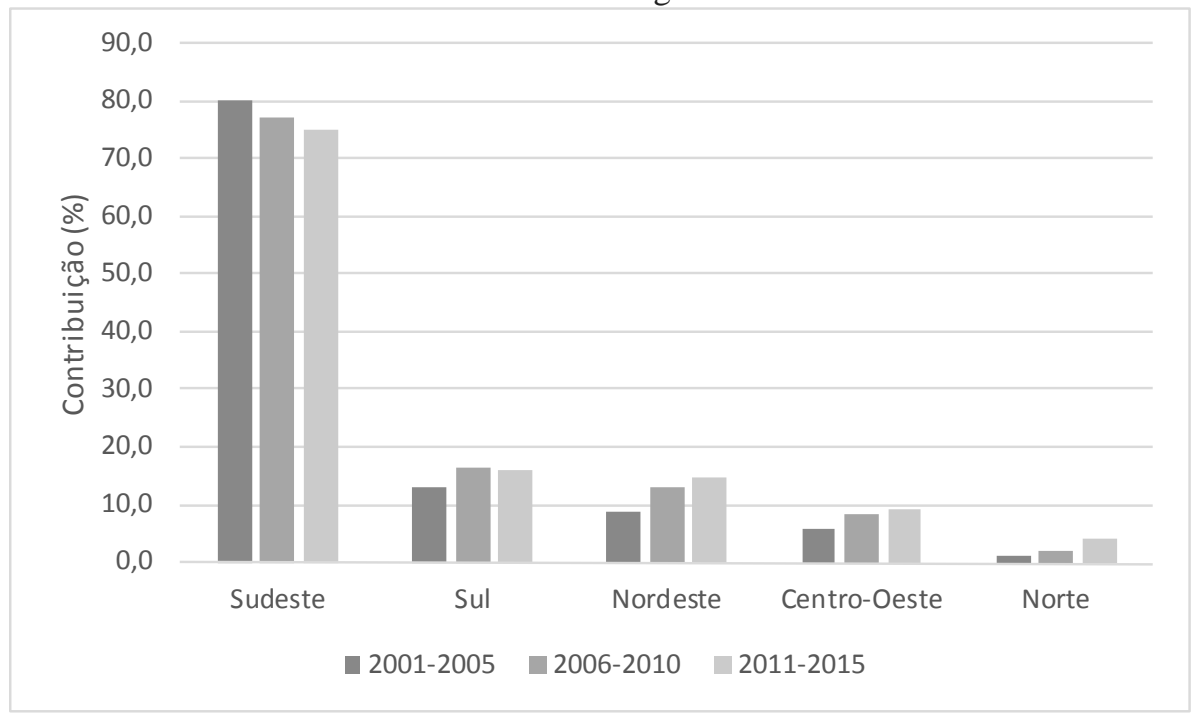

Fonte: Dados da pesquisa.

No período entre 2001 e 2005, 46,2\% das publicações da região nordeste envolveram apenas pesquisadores da região, conforme Figura 4, o que caracteriza que nessas publicações não houve colaboração de pesquisadores do nordeste com outros das demais regiões do país. Esse percentual de publicações sem colaborações, no entanto, decresceu nos quinquênios seguintes, com o aumento do número de publicações em parceria com instituições nacionais localizadas em outras regiões geográficas do Brasil, principalmente, São Paulo, Minas Gerais, Rio de Janeiro e Rio Grande do Sul. Já o percentual de publicações científicas em colaboração com instituições do exterior permaneceu relativamente constante nos períodos analisados, em torno dos $30 \%$, em patamar semelhante ao indicado pela literatura para a colaboração internacional do Brasil, em todas as áreas, no período entre 2002 e 2006, que foi de 31\%.

O avanço das pesquisas sobre Biotecnologia no nordeste tem forte conexão com as pesquisas realizadas no Sudeste brasileiro, conforme apresentado na Figura 5, em que o tamanho de cada nó é proporcional ao total de publicações de cada Estado ou Região, no período de 2001 a 2015, e a espessura das linhas é proporcional ao número de publicações em colaboração. Embora haja colaboração científica entre os Estados do nordeste, com proeminência de Pernambuco, do Ceará e da Bahia, como mostrado na Figura 5, 
a colaboração é mais intensa com instituições localizadas no Sudeste do país. Neste sentido, o Centro-Oeste também desempenha um papel significativo em conjunto com a região do nordeste. Essa colaboração pode estar associada ao fato da região Sudeste concentrar um grande número de universidades e institutos de pesquisa que também atuam no tema. Outro ponto é que foi constatado, ainda em 2002, que as regiões Sudeste e Sul do país concentravam naquela época 90\% das empresas atuantes em Biotecnologia, enquanto que o Norte e o nordeste contavam com 3\% dessas empresas, o que pode ter estimulado as parcerias (CENTRO DE GESTÃO E ESTUDOS ESTRATÉGICOS, 2002).

Figura 4 - Percentual de publicações do nordeste sem colaboração e com colaboração com instituições internacionais e nacionais de outras regiões brasileiras em diferentes quinquênios

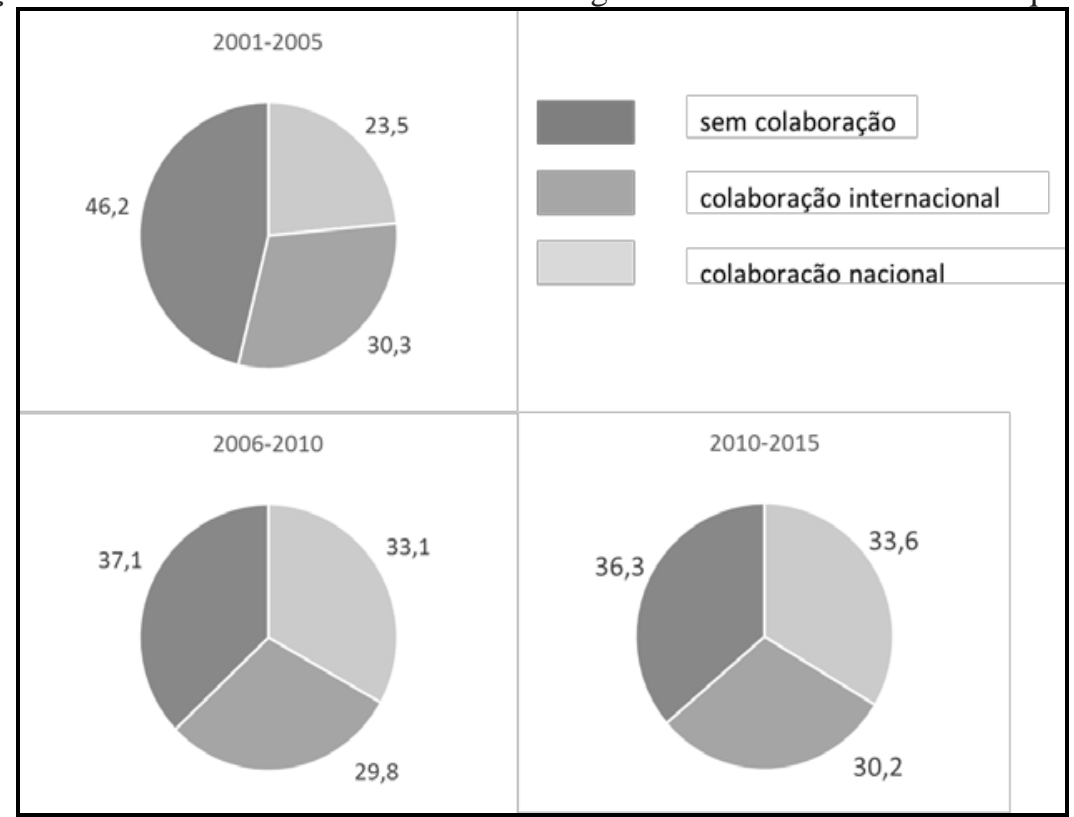

Fonte: Dados da pesquisa.

Além disso, é importante salientar que pouco a pouco as bases científicas e tecnológicas do nordeste têm sido transformadas pelas políticas nacionais de Ciência, Tecnologia e Inovação orientadas à redução das desigualdades regionais (CENTRO DE GESTÃO E ESTUDOS ESTRATÉGICOS, 2014, 2016), e espera-se que esse fato tenha impacto direto no aumento da produção científica da região, em todas as áreas. Observou-se um expressivo processo de expansão, fortalecimento e criação de novas universidades, instituições de 
ensino superior, institutos de pesquisa e de ensino tecnológico, além de laboratórios especializados no nordeste.

Figura 5 - Rede de colaboração entre os estados do nordeste e outras regiões brasileiras

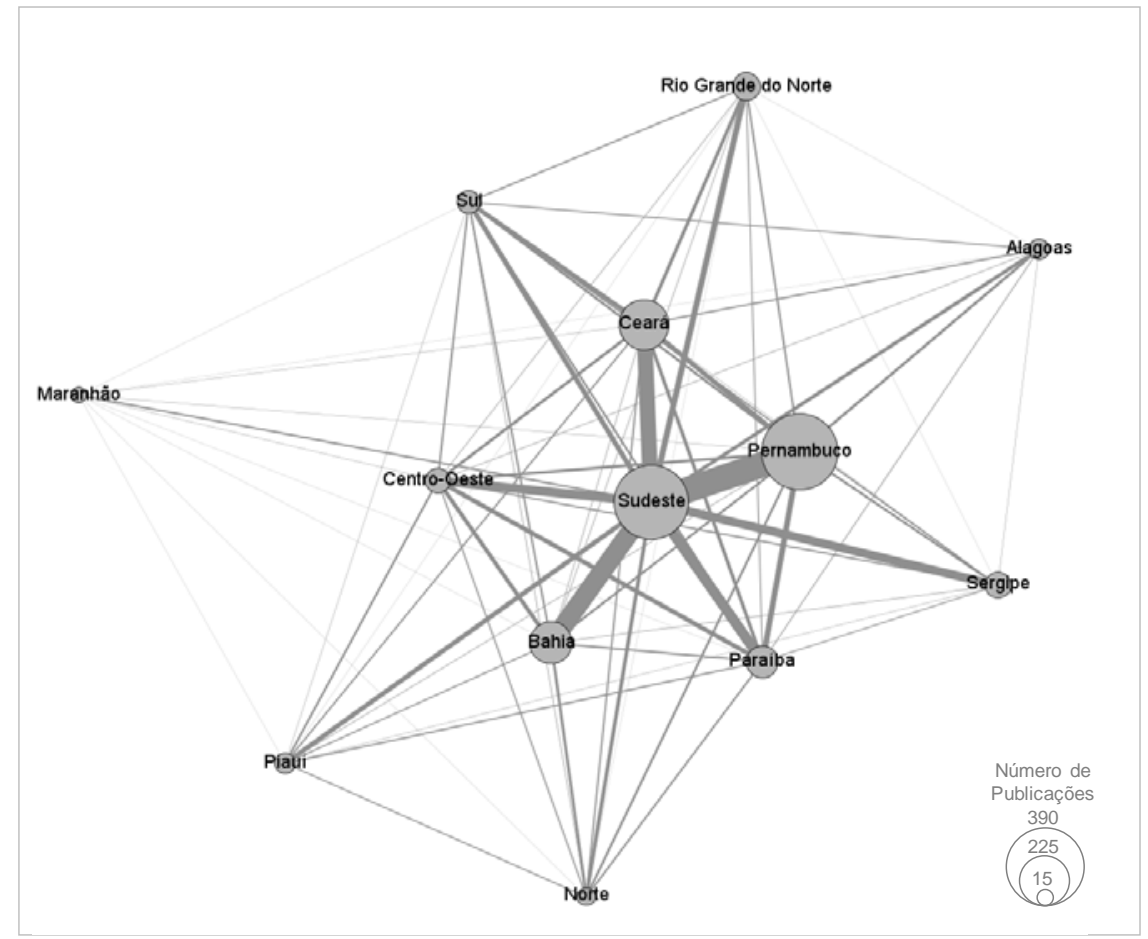

Fonte: Dados da pesquisa.

\subsection{Papel da RENORBIO nos avanços científicos em Biotecnologia}

As instituições integrantes da RENORBIO têm cumprido seu papel no que diz respeito ao avanço científico em Biotecnologia. Em relação à produção científica do nordeste, a RENORBIO é responsável por, aproximadamente, 90\% das publicações da região, conforme mostrado na Figura 6, independente do período considerado. Consequentemente, as tendências observadas para a região nordeste, como aumento da colaboração com instituições nacionais (Figura 4) e a forte dependência da região Sudeste (Figura 5), são resultados, pelo menos em parte, da atuação da RENORBIO. É notória também a contribuição ascendente da RENORBIO nas pesquisas em Biotecnologia desenvolvidas no Brasil, atingindo 13,0\% no quinquênio 2011-2015, e com tendência de crescimento nos próximos anos.

A RENORBIO foi oficialmente criada em 2003. Suas instituições integrantes têm aumentado a colaboração entre si, conforme se observa no 
conjunto de redes de colaboração apresentadas nas Figuras 7, 8 e 9, nas quais o tamanho dos nós é proporcional ao total de publicações de cada instituição e a espessura das linhas é proporcional ao número de publicações em colaboração entre as instituições ligadas. Num primeiro momento, apenas 18 instituições tiveram publicações em Biotecnologia em periódicos específicos ao tema indexados na Web of Science (Figura 7), porém esse número passou para 30 no último quinquênio (Figura 9), indicando o engajamento de outras instituições da região. Houve também aumento na densidade da rede de colaboração conforme os períodos, o que indica a consolidação da RENORBIO devido à maior coesão observada na rede, embora haja espaço para mais colaborações, já que o valor encontrado para o período 2011-2015 está relativamente longe de 1 (limite de colaboração, quando todos os elementos da rede estão conectados entre si).

Figura 6 - Contribuição das pesquisas realizada por instituições integrantes da RENORBIO para as pesquisas em Biotecnologia realizadas no nordeste e no Brasil

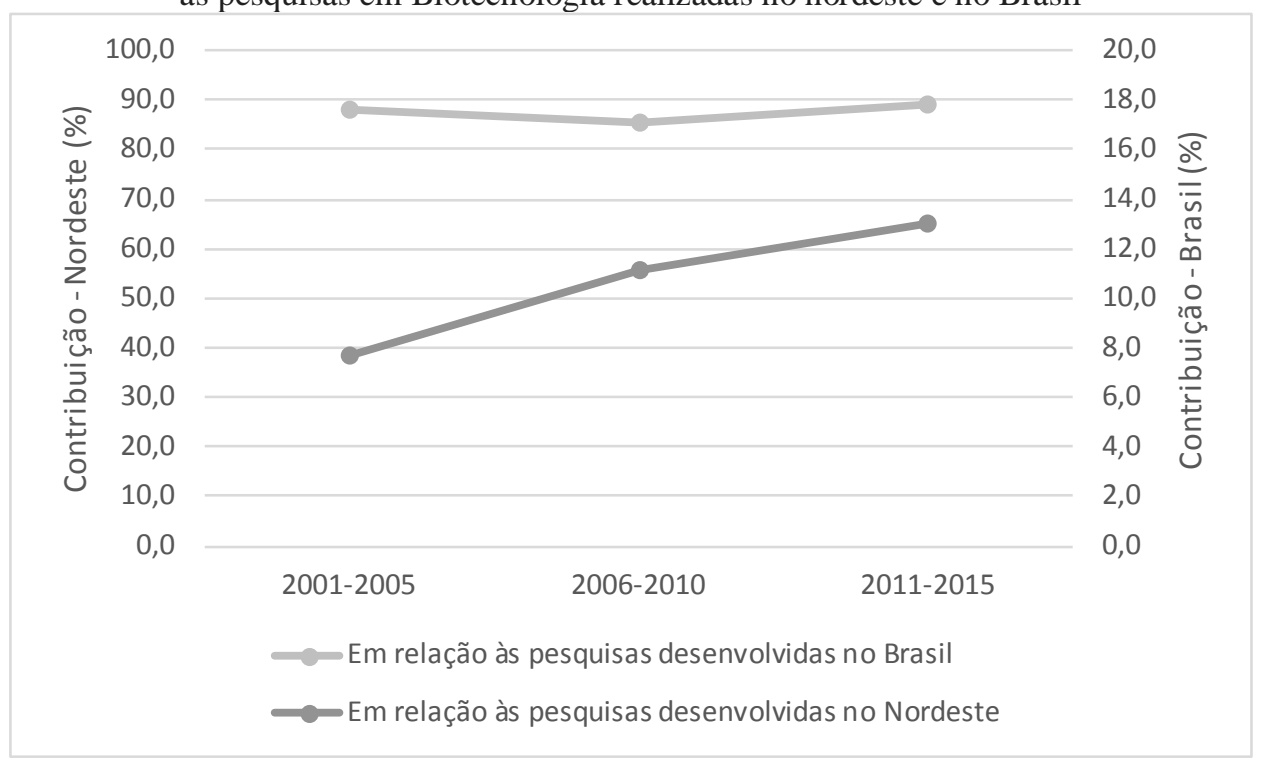

Fonte: Dados da pesquisa. 
Figura 7 - Rede de colaboração entre as instituições integrantes da RENORBIO em 2001-2005

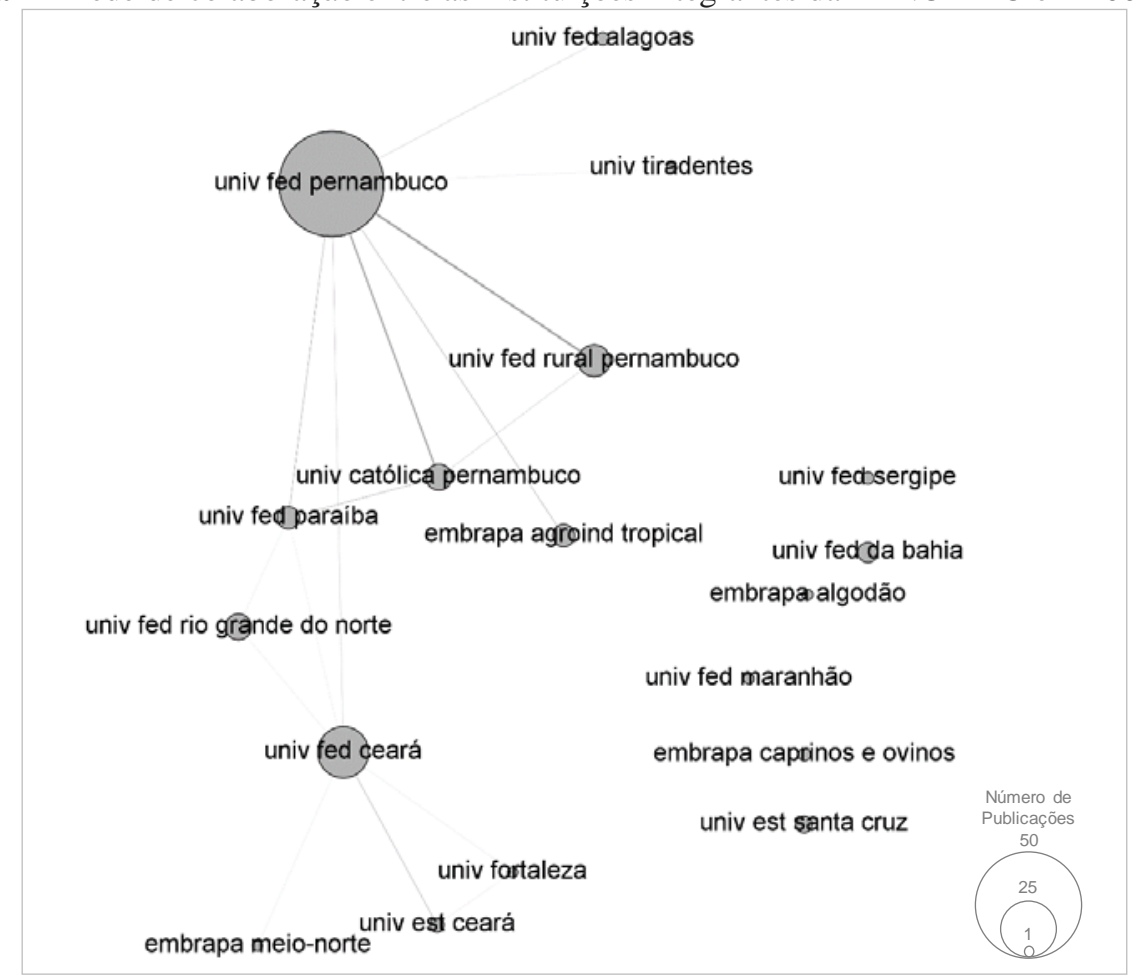

Fonte: Dados da pesquisa.

Nota: Número de Elementos: 18; Densidade da rede: 0,105.

Figura 8 - Rede de colaboração entre as instituições integrantes da RENORBIO em 2006-2010

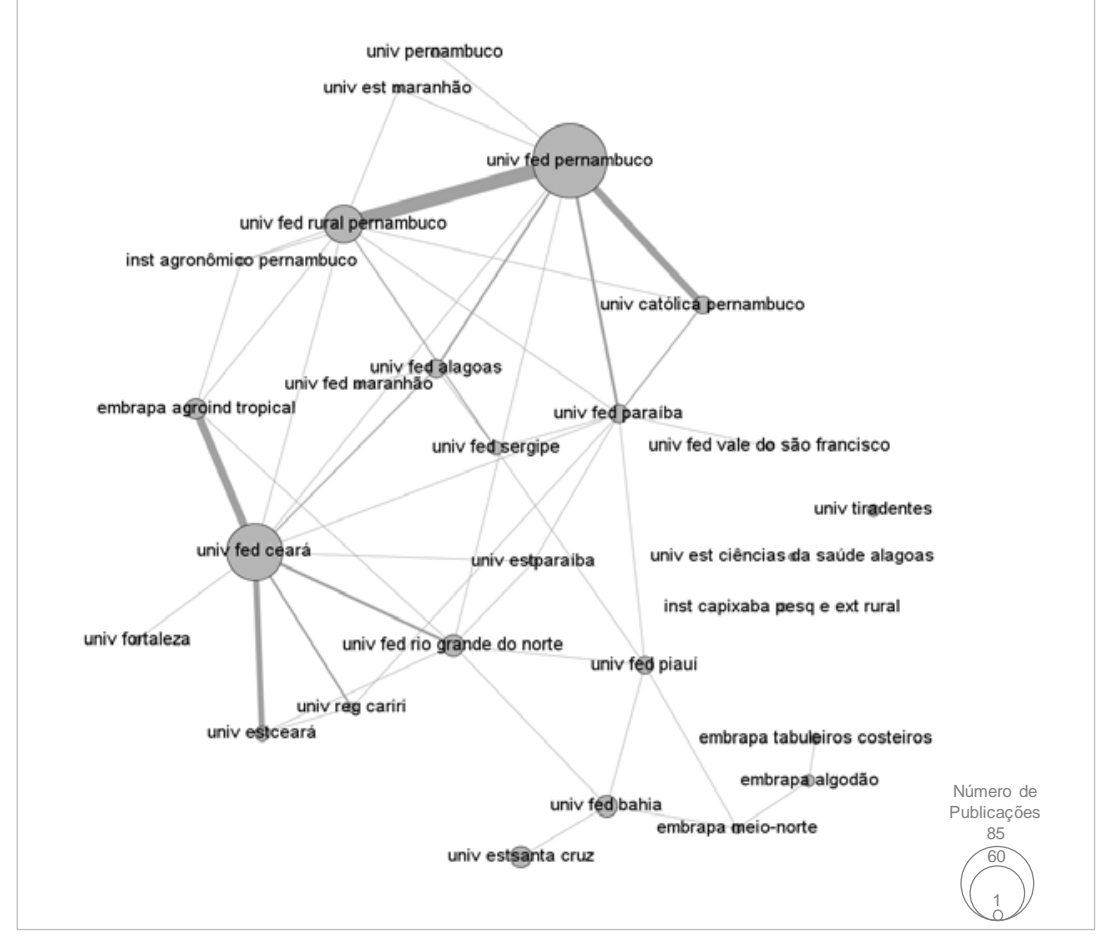

Fonte: Dados da pesquisa.

Nota: Número de elementos: 27; Densidade da rede: 0,13. 
Figura 9 - Rede de colaboração entre as instituições integrantes da RENORBIO em 2011-2015

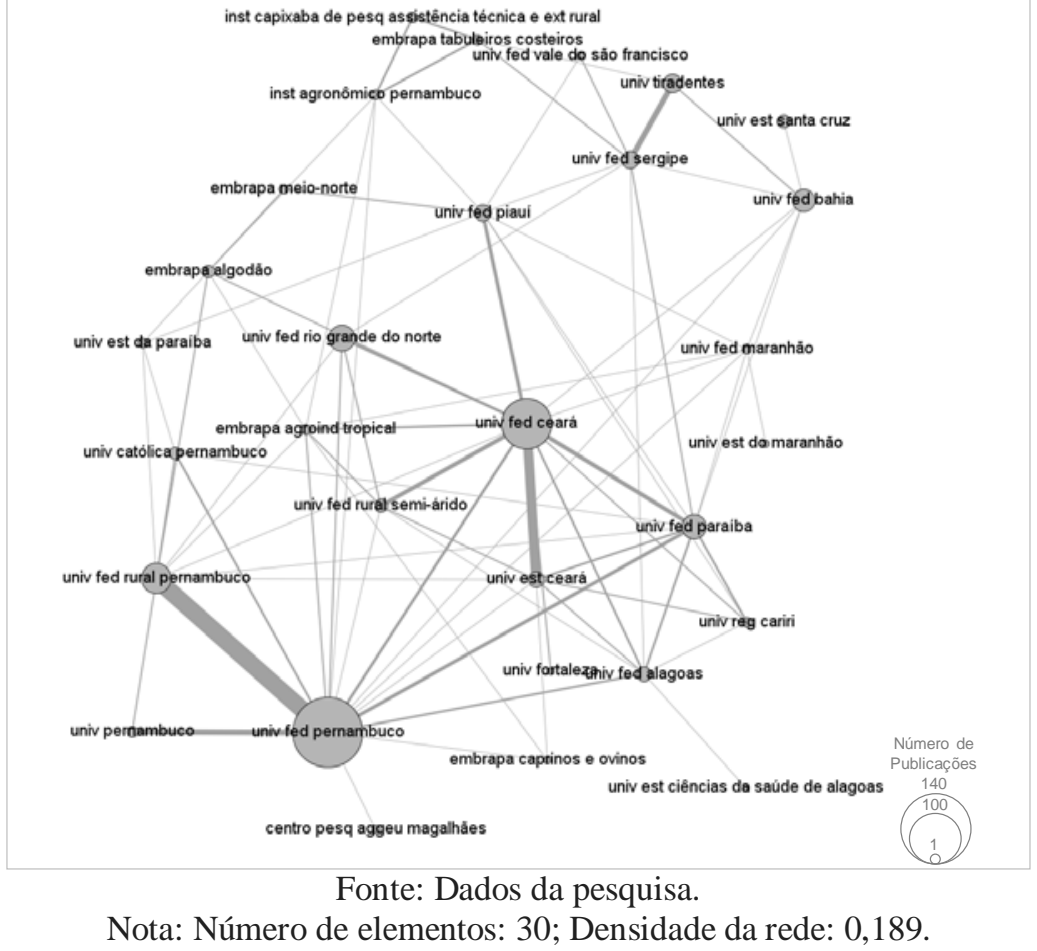

No período entre 2001 e 2005, a Universidade Federal do Pernambuco e a Universidade Federal do Ceará lideraram o desenvolvimento das pesquisas em Biotecnologia, conforme Figura 7. Embora estas duas universidades também tenham claramente um papel central nas redes das Figuras 8 e 9 e, portanto, nas pesquisas em Biotecnologia da RENORBIO, houve ascensão de outras instituições, em especial no quinquênio 2010-2015. São elas: a Universidade Federal da Paraíba, a Universidade Federal Rural de Pernambuco, a Universidade Federal do Maranhão e a Embrapa Agroindústria Tropical. Tais instituições, apesar do menor número acumulado de publicações nos quinquênios em relação às líderes, passaram a ter uma relevância maior na rede de colaboração, por conectarem outros elementos à rede principal.

\section{Conclusão}

O Brasil tem contribuído cada vez mais para as pesquisas mundiais em Biotecnologia. O crescimento da produção científica nacional nesse assunto em termos absolutos e também quando considerada a contribuição do país para a produção científica internacional, evidencia que as instituições de ciência e 
tecnologia estão em sintonia com as diretrizes de pesquisa e desenvolvimento expressas pelo governo através das políticas públicas de ciência e tecnologia.

As instituições de pesquisa localizadas na Região Sudeste do país ainda respondem pela maior parte da produção científica nacional em Biotecnologia.

No entanto, a contribuição de instituições dessa região para o total da produção científica nacional tem caído com o passar do tempo, para os três períodos analisados. Esse comportamento é semelhante ao verificado para toda a produção científica nacional, independente de tema.

A região nordeste apresentou crescimento na contribuição para a produção científica nacional no tema, sendo a RENORBIO uma iniciativa importante nesse cenário, consolidando-se como uma rede de colaboração para promoção das pesquisas. Nesse sentido, destaca-se seu papel preponderante para a produção científica da região nordeste e uma contribuição ascendente em relação às pesquisas desenvolvidas no âmbito nacional, como provável consequência do aumento da colaboração científica entre as instituições que a integram nos últimos anos. Por exemplo, a partir de 2006, uma maior proporção de artigos em colaboração nacional ou internacional foi identificada, indicando uma maior participação da região nordeste no debate científico sobre Biotecnologia.

Conclui-se que a implementação de políticas públicas em ciência e tecnologia visando à redução das desigualdades regionais tem surtido efeito na área, como indicado pelo aumento, ainda que pequeno, da contribuição da região do nordeste na produção científica brasileira, no período de 2001 a 2015. Conclui-se também, que a formação de redes colaborativas de pesquisa pode ser um instrumento poderoso para alavancar as atividades de pesquisa em uma região e/ou tema específicos e seus resultados.

\section{Agradecimentos}

Os autores agradecem os recursos da FUNCAP que contribuíram para a realização deste trabalho. 


\section{Referências}

BRASIL. Ministério da Saúde. Tesauro Eletrônico. Biotecnologia. 2016a.

Disponível em: <http://bvsms2.saude.gov.br/cgi-

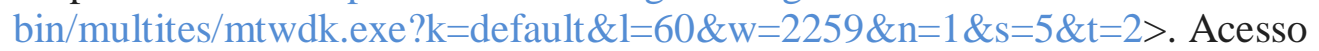

em: 26 fev. 2016.

BRASIL. Lei no 10.332, de 19 de dezembro de 2001. Diário Oficial [da]

União, Brasília, DF, 20 dez. 2001a. Disponível em:

<http://www.planalto.gov.br/ccivil_03/leis/LEIS_2001/L10332.htm>. Acesso em: 26 fev. 2016.

BRASIL. Lei $\mathrm{n}^{\circ}$ 11.105, de 24 de março de 2005. Diário Oficial [da] União, Brasília, DF, 28 mar. 2005. Disponível em:

<http://www.planalto.gov.br/ccivil_03/_ato2004-2006/2005/lei/l11105.htm>. Acesso em: 4 mar. 2016.

BRASIL. Lei ${ }^{\circ}$ 13.123, de 20 de maio de 2015. Diário Oficial [da] União, Brasília, DF, 21 maio 2015. Disponível em:

<http://pesquisa.in.gov.br/imprensa/jsp/visualiza/index.jsp?jornal=1 \&pagina=1 \&data=21/05/2015>. Acesso em 14 abr. 2016.

BRASIL. Medida provisória n 2.186-16, de 23 de agosto de 2001. Diário Oficial [d]a União, Brasília, DF, 24 ago. 2001b. Disponível em:

<http://pesquisa.in.gov.br/imprensa/jsp/visualiza/index.jsp?jornal=1\&pagina=11 \&data=24/08/2001>. Acesso em: 14 abr. 2016.

BRASIL. Ministério da Ciência, Tecnologia e Inovação. Estratégia nacional de ciência, tecnologia e inovação 2012 - 2015. Brasília, DF, 2012. Disponível em: <http://www.mct.gov.br/upd_blob/0218/218981.pdf>. Acesso em: 13 abr. 2016.

BRASIL. Ministério do Planejamento, Orçamento e Gestão. PPA 2012-2015. 2016a. Disponível em:

<http://www.planejamento.gov.br/assuntos/planejamento-einvestimentos/plano-plurianual>. Acesso em: 4 mar. 2016.

BRASIL. Ministério da Ciência, Tecnologia e Inovação. Proposta da estratégia nacional de ciência, tecnologia e inovação 2016-2019. Brasília, DF, 2016b. Disponível em:

<http://www.mcti.gov.br/documents/10179/35540/Estrat\%C3\%A9gia+Nacional + de+Ci\%C3\%AAncia\%2C\%20Tecnologia+e+Inova\%C3\%A7\%C3\%A3o+\%28 Encti\%29\%202016-2019+-+documento+para+discuss\%C3\%A3o/5a4fe994955e-4658-a53c-bc598af09f7e>. Acesso em: 13 abr. 2016.

CENTRO DE GESTÃO E ESTUDOS ESTRATÉGICOS. Descentralização do fomento à ciência, tecnologia e inovação no Brasil. 2010. Disponível em: <www.cgee.org.br/atividades/redirect.php?idProduto=6402>. Acesso em: 4 mar. 2016. 


\section{CENTRO DE GESTÃO E ESTUDOS ESTRATÉGICOS. Diretrizes} estratégicas do Fundo Setorial de Biotecnologia. 2002. Disponível em: $<$ https://www.finep.gov.br/images/a-finep/fontes-de-orcamento/fundossetoriais/ct-bio/diretrizes-estrategicas-para-o-fundo-setorial-debiotecnologia.pdf>. Acesso em: 4 mar. 2016.

CENTRO DE GESTÃO E ESTUDOS ESTRATÉGICOS. Plano de ciência, tecnologia e inovação para desenvolvimento sustentável do nordeste brasileiro. 2014. Disponível em: <www.cgee.org.br/atividades/redirect/8936>. Acesso em: 10 jun. 2016.

CENTRO DE GESTÃO E ESTUDOS ESTRATÉGICOS. The brazilian innovation system: a mission-oriented policy proposal. 2016. Disponível em: $<$ https://www.cgee.org.br/the-brazilian-innovation-system>. Acesso em: 4 mar. 2016.

DALPÉ, R. Bibliometric analysis of biotechnology. Scientometrics, Dordrecht, v. 55, n. 2, p. 189-213, 2002. Disponível em:

<http://dx.doi.org/10.1023/A:1019663607103>. Acesso em: 14 abr. 2016.

DAVYT, A.; VELHO, L. A avaliação da ciência e a revisão por pares: passado e presente. Como será o futuro? História, Ciências, Saúde: Manguinhos, Rio de Janeiro, v. 2, n. 1, p. 93-116, 2000.

FARIA, L. I. L. de et al. Análise da produção científica a partir de publicações em periódicos especializados. In: FUNDAÇÃO DE AMPARO À PESQUISA DO ESTADO DE SÃO PAULO. Indicadores de ciência, tecnologia e inovação em São Paulo 2010. São Paulo, 2010.

FREEMAN, C.; SOETE L. Developing science, technology and innovation indicators: what we can learn from the past. Research Policy, Amsterdam, v. 38, n. 4, p. 583-589, 2009. Disponível em:

<http://www.sciencedirect.com/science/article/pii/S0048733309000237>. Acesso em: 14 abr. 2016.

GLANZEL, W. et al. Biotechnology: an analysis based on publications and patents. 2003. Disponível em: <http://www.steunpuntoos.be>. Acesso em: 14 abr. 2016.

GOKHBERG, L. Indicators for science, technology and innovation on the crossroad to foresight. In: MEISSNER, D.; GOKHBERG, L.; SOKOLOV, A. (Ed.). Science, technology and innovation policy for the future. Heidelberg: Springer-Verlag, 2013. p. 257-288. Disponível em:

<http://dx.doi.org/10.1007/978-3-642-31827-6_15>. Acesso em: 14 abr. 2016.

GREGOLIN, J. A. R. et al. Análise da produção científica a partir de indicadores bibliométricos. In: FUNDAÇÃO DE AMPARO À PESQUISA DO 
ESTADO DE SÃO PAULO. Indicadores de ciência, tecnologia e inovação em São Paulo 2004. São Paulo, 2005. p. 1-44.

HUANG, M. H.; YANG, H. W.; CHEN, D. Z. Increasing science and technology linkage in fuel cells: a cross citation analysis of papers and patents. Journal of Informetrics, [S.I.], v. 9, n. 2, p. 237-249, 2015. Disponível em: <http://dx.doi.org/10.1016/j.joi.2015.02.001>. Acesso em: 20 mar. 2016.

MOED, H. F.; GLÄNZEL, W.; SCHMOCH, U. (Ed.). Handbook of quantitative science and technology research: the use of publication and patent statistics in studies of S\&T systems. New York: Kluwer Academic Publishers, 2004.

MOURA, A. M. M. Motivação para a pesquisa, determinação de parcerias e divisão da coautoria e coinvenção: principais critérios utilizados pelos pesquisadores da área da biotecnologia. Em Questão, Porto Alegre, v. 18, n. 3. Edição Especial, p. 29-45, dez. 2012. Disponível em:

<http://seer.ufrgs.br/index.php/EmQuestao/article/view/33025>. Acesso em: 15 maio 2016.

MOURA, A. M. M.; CAREGNATO, S. E. Co-classificação entre artigos e patentes: um estudo da interação entre C\&T na biotecnologia brasileira.

Informação \& Sociedade, João Pessoa, v. 20, n. 2, p. 119-132, 2010.

Disponível em:

<http://www.ies.ufpb.br/ojs2/index.php/ies/article/view/3757/4362>. Acesso em: 15 maio 2016.

OKUBO, Y. Bibliometric indicators and analysis of research systems: methods and examples. 1997. Disponível em: <http://www.oecdilibrary.org/science-and-technology/bibliometric-indicators-and-analysis-ofresearch-systems_208277770603>. Acesso em: 12 abr. 2016.

ORGANISATION FOR ECONOMIC CO-OPERATION AND DEVELOPMENT (OECD). Glossary of statistical terms. Paris, 2007.

Disponível em: <http://stats.oecd.org/glossary/detail.asp?ID=198>. Acesso em: 12 abr. $/ 2016$.

PAYUMO, J. G.; SUTTON, T. C. A bibliometric assessment of ASEAN collaboration in plant biotechnology. Scientometrics, Dordrecht, v. 103, n. 3, p. 1043-1059, 2015. Disponível em: <http://dx.doi.org/10.1007/s11192-015-1582x>. Acesso em: 20 abr. 2016.

RENORBIO. Histórico: conheça melhor a história da Renorbio. 2016. Disponível em: <http://www.renorbio.org.br/portal/renorbio/historico.htm>. Acesso em: 12 abr. 2016.

SPINAK, E. Indicadores cienciometricos. Ciência da Informação, Brasília, DF, v. 27, n. 2, p. 141-148, 1998. 
VANTI, N. A. P. Da bibliometria à webometria: uma exploração conceitual dos mecanismos utilizados para medir o registro da informação e a difusão do conhecimento. Ciência da Informação, Brasília, DF, v. 31, n. 2, p. 152-162, 2002.

VELHO, L.; SOUZA-PAULA, M. C. Introdução. In: CENTRO DE GESTÃO E ESTUDOS ESTRATÉGICOS. Avaliação de políticas de ciência, tecnologia e inovação: diálogo entre experiências internacionais e brasileiras. Brasília, DF, 2008. p. 9-18.

\title{
Bibliometric analysis of Brazilian and northeast Brazilian region scientific publication in biotechnology
}

\begin{abstract}
Biotechnology consolidates itself as a theme that has been repeatedly considered as strategic subject for the development of Brazil. Therefore, there is a need to investigate the impact of public policies on science and technology, such as the pluriannual plans and the National Science and Technology Strategy, which aim to stimulate scientific research in Biotechnology. The main goal of this article was to elaborate and analyze a set of bibliometric indicators on scientific production in Biotechnology. The method included the use of bibliometrics as an information analysis technique and the analyzed sample contained 517,569 bibliographic records referring to the scientific production on Biotechnology indexed in the Web of Science database, from 2001 to 2015. The results achieved included indicators of production and national and international scientific collaboration and point to the increase of Brazil's contribution to the world scientific production in Biotechnology, as well as the growing importance of research carried out by the institutions of the Northeast Region of Brazil, as well as the collaboration among them. It is concluded that the implementation of public policies in science and technology aimed at reducing regional inequalities have had an effect in the Biotechnology area.
\end{abstract}

Keywords: Biotechnology. Science \& Technology Indicators. RENORBIO. Bibliometrics.

Recebido em: 29/11/2016

Aceito em: 23/01/2017 